\title{
Interoperable End-to-End Remote Patient Monitoring Platform Based on IEEE 11073 PHD and ZigBee Health Care Profile
}

\author{
Malcolm Clarke ${ }^{(1)}$, Senior Member, IEEE, Joost de Folter, Vivek Verma, and Hulya Gokalp
}

\begin{abstract}
This paper describes the implementation of an end-to-end remote monitoring platform based on the IEEE 11073 standards for personal health devices (PHD). It provides an overview of the concepts and approaches and describes how the standard has been optimized for small devices with limited resources of processor, memory, and power that use short-range wireless technology. It explains aspects of IEEE 11073, including the domain information model, state model, and nomenclature, and how these support its plug-and-play architecture. It shows how these aspects underpin a much larger ecosystem of interoperable devices and systems that include IHE PCD-01, HL7, and BlueTooth LE medical devices, and the relationship to the Continua Guidelines, advocating the adoption of data standards and nomenclature to support semantic interoperability between health and ambient assisted living in future platforms. The paper further describes the adaptions that have been made in order to implement the standard on the ZigBee Health Care Profile and the experiences of implementing an end-to-end platform that has been deployed to frail elderly patients with chronic disease(s) and patients with diabetes.
\end{abstract}

Index Terms-Ambient assisted living, continua Health Guidelines, chronic disease, IEEE 11073, IHE PCD-01, integrated care, interoperability, remote patient monitoring, standards based platform, telehealth, telecare, well-being, zigbee Health Care Profile.

\section{INTRODUCTION}

$\mathbf{R}$ EMOTE patient monitoring platforms are deployed to patients in order to collect and forward observations to the healthcare professional so that changes in the state of health might be determined. Current applications typically monitor and manage patients with chronic disease with the purpose of controlling costs as well as clinical outcomes [1]. Use is often restricted to patients with congestive heart failure (CHF) or chronic obstructive pulmonary disease (COPD), as patients with these conditions are known to consume significant health resources, which might be reduced by remote patient monitoring [2].

Manuscript received February 1, 2017; revised April 9, 2017; accepted July 23, 2017. Date of publication August 9, 2017; date of current version April 19, 2018. This work was supported by the EPSRC under Grant TS/H000100/1. (Corresponding author: Malcolm Clarke.)

M. Clarke is with the Computer Science Department, Brunel University, Uxbridge UB8 3PH, U.K. (e-mail: malcolm.clarke @ brunel.ac.uk).

J. de Folter, V. Verma, and H. Gokalp are with the Computer Science Department, Brunel University.

Digital Object Identifier 10.1109/TBME.2017.2732501
Platforms have become specific to these needs and so have remained largely unchanged since the introduction of the first commercial devices. This included the Care Companion by American Medical Devices, with a restricted number of sensors to monitor physiological parameters including blood pressure, weight, blood glucose and $\mathrm{SpO}_{2}$ [3]. Platforms were developed to include provision for simple question and answer (e.g., symptom data) and manual entry of values, when specific devices were not available to the platform, typified by the Health Hero (Bosch). However, each sensor was based on its own proprietary protocol that made it impossible to add further types of sensor to a system without an update to the firmware, resulting in systems that remained limited in functionality and based only on the same common device types. Even recent commercial offerings such as Health Hero (Bosch), Ideal Life, and Motiva (Philips) continue to include only a limited set of sensors based on proprietary protocols, resulting in functionality of the system remaining as restricted as before.

Recently, researchers have reported approaches based on standards such as IEEE 11073 [4] to achieve plug-and-play interoperability. However, early efforts were hampered by being based on draft standards, overly complex protocols designed to work with large intensive care devices, lack of well-developed forms of wireless technology and limitations imposed by the embedded processors available at that time. As a result, implementation was compromised; simplifications were made and a fixed set of messages were used in place of a generic approach, prohibiting simple extension to new types of device, as is the intention of the protocol.

Moreover, these early efforts failed to consider the design of the architecture of the complete system in order to achieve endto-end plug-and-play interoperability, not only from the sensor to the local gateway, but also from gateway to the enterprise. Implementations continued to use proprietary communication protocols, and did not consider how to exploit standards based protocols such as IHE PCD-01 (a profile of HL7) [5], [6] to forward observations to the remote database in the enterprise using a transparent mapping from one protocol to the other.

The situation remains and most research projects on remote patient monitoring platforms largely continue to integrate sensors and transmit data to the gateway using proprietary protocols and transmit the data from the gateway using further proprietary protocols. Despite projects exploiting convergence of lower layer protocols such as web services, Internet of Things 


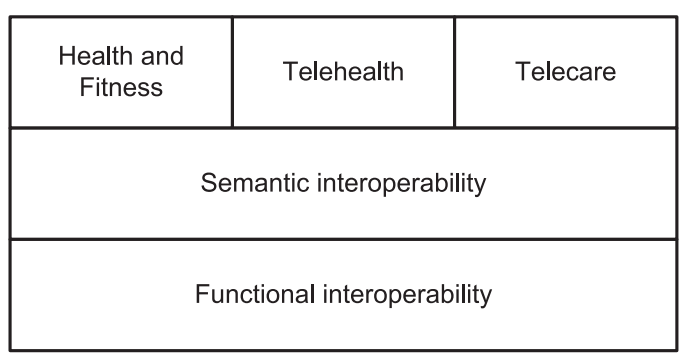

Fig. 1. Semantic operability across domains.

(IoT) [7] and cloud computing [8], together with presentation protocols such as XML and JSON, they do not include consideration of higher layer protocols, data models, nomenclature, extensibility, or semantic interoperability, and suggest that translation may suffice, despite the well-known problems of mapping between existing schemes such IEEE 11073-10101 and LOINC.

\section{IEEE 11073 Baseline Standards For Personal HEALTH DEVICES (PHD)}

\section{A. Interoperability Across Telemedicine Domains}

The IEEE 11073 Personal Health Devices (PHD) initiative was designed to take the base standards of the original IEEE 11073 family and develop them to match the capabilities of low power embedded devices and ultra-low power wireless technologies. The protocol in some layers, such as session, was much simplified or removed; other aspects were made more efficient and the interface to an underlying transport layer clearly defined. Work was then undertaken to define transport technologies to match this interface, resulting in the base protocol IEEE 11073-20601 [9]. BlueTooth, USB and ZigBee each developed a standard (NFC more recently), and these are profiled by the guidelines of the Continua Alliance [10] to ensure interoperability and to provide for testing of compliance. At the same time, the IEEE 11073 PHD committee produced an accompanying set of specialization standards for the devices, which included disease management (alternatively called telehealth), health and fitness, and independent living (alternatively called telecare, ambient assisted living (AAL), activities of daily living (ADL)), that would ensure semantic interoperability across these separate domains (see Fig. 1).

The goal of semantic interoperability between telehealth and telecare may be accomplished using the standards that have been defined by IEEE 11073 and the transport technologies as shown in Fig. 2. At the same time, the standards ensure that devices are interoperable with devices of different transport technologies, both semantically and at protocol (application protocol data unit (APDU) level).

Currently USB and BlueTooth (BT) Health Device Profile (HDP) only support devices from the telehealth domain due to limitations of range, power and the number of devices that can be connected concurrently.

However ZigBee (ZigBee Health Care Profile) has the characteristics (range, power and concurrently connected devices) to support devices from both the telehealth and telecare

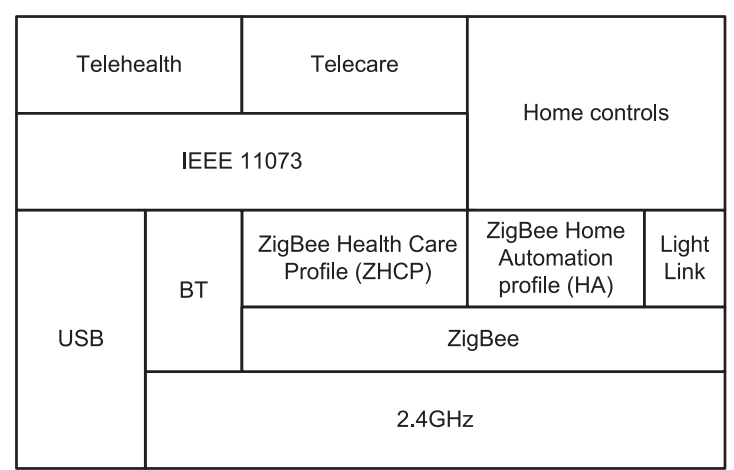

Fig. 2. Standards supporting interoperability across domains.

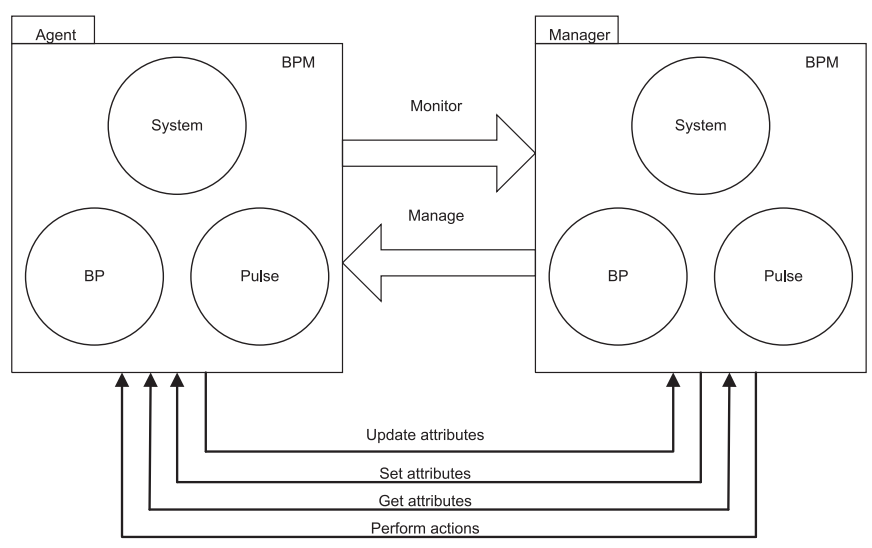

Fig. 3. Generic IEEE 11073 object model.

domains on a single wireless technology. Moreover, by adopting a standard for ZigBee, the other profiles of the underlying ZigBee protocol including Home Automation (HA) and Light Link might be used to bridge between the IEEE 11073 domain and other domains, such as home controls, in the home environment to provide increased functionality for the in-home gateway (e.g., controlling lights on exit/entry to bed) (see Fig. 2).

\section{B. The Generic IEEE 11073 Object Model}

IEEE 11073-20601 [9] employs a generic object model (referred as the Domain Information Model - DIM) as its approach to manage and monitor medical devices. The system (referred as the Medical Device System (MDS)) and each data source are modelled as separate objects in the agent (see Fig. 3). Proxy copies of the objects are created in the manager on association and the values of attributes are updated using a restricted set of basic methods that operate on each of the objects in welldefined ways. Changes to the value of any attribute in the agent are notified to the manager by an event, which would include any observation taken by agent.

In theory, several approaches can be used to construct a copy of the objects and attributes of the agent in the manager, and fundamentally each technique achieves the same result, although each approach has advantages and disadvantages. One approach is to retrieve all the attributes of all the objects using the GET method, where the value of an attribute is returned in response to a request. This approach is employed in several technologies, 
TABLE I

TYPICAL MDS OBJECT ATTRIBUTES

\begin{tabular}{lll}
\hline \hline Attribute name & \multicolumn{1}{c}{ Remark } & \multicolumn{1}{c}{ Value } \\
\hline Handle & $\begin{array}{l}\text { A unique reference ID } \\
\text { for this object }\end{array}$ & 0 \\
\hline System-Type-Spec-List & $\begin{array}{l}\text { The type and version of } \\
\text { the agent }\end{array}$ & $\begin{array}{l}\text { \{MDC_DEC_SPEC_- } \\
\text { PROFILE_SCALE,1 }\end{array}$ \\
\hline System-Model & Manufacturer and model & "Brunel","SCALE" \\
\hline System-Id & $\begin{array}{l}\text { Unique ID for the device } \\
\text { - IEEE EUI-64 }\end{array}$ & 8016B7123456789A \\
\hline Dev-Configuration-Id & $\begin{array}{l}\text { Identifies the } \\
\text { configuration of the } \\
\text { device. }\end{array}$ & 1500 \\
\hline Date-and-Time & Current time of the agent & $21 / 11 / 2015-11: 35: 24.44$ \\
\hline MDS-Time-Info & $\begin{array}{l}\text { Defines capabilities and } \\
\text { status of the time in the } \\
\text { device }\end{array}$ & $\begin{array}{l}\text { Accuracy, "set please", } \\
\text { time format(s) } \\
\text { supported, synchronized }\end{array}$ \\
\hline Power-Status & Mains/battery & Battery \\
\hline Battery-Level & Battery level in \% & 75 \\
\hline \hline
\end{tabular}

such as BlueTooth Low Energy (LE). However the technique has a number of disadvantages. The greatest disadvantage is that the manager must still be informed of changes to any attribute in the agent and an update method to notify these events is required; alternatively the manager reads all relevant attributes (e.g., unit) when an observation is received to ensure all are up to date. For a small number of observations this can minimize the overall size of the transmission, as sending the full configuration of the agent can be avoided.

The approach of IEEE 11073 has the advantage that transmission is minimized when many observations are sent, as only changes (e.g., the observation and its timestamp) are sent; all other attributes are up to date and are not required to be sent in the observation. However note that IEEE 11073-20601 introduces an optimized association protocol that obviates the need to send the full configuration on every association (see section III.F for full details of the optimizations).

\section{Restricted Classes for Agent Modeling}

The approach of IEEE 11073 to the modelling of an agent is to use a consistent and restricted set of generic classes and use attributes to provide the context (type) for each object. This is in contrast to the approach where bespoke classes (or messages) are defined for each type of device.

The advantages are that: the programming requirements for agent and manager are constrained; classes are reused; generic managers can be realized; and no firmware upgrades are required in a manager to support a new type of device.

An agent will always have an MDS object, with typical attributes and values as in Table I. The MDS provides system level information and status. Typically the attributes of the MDS object are retrieved once on association as most attribute values are static. There may be a periodic check on the Date-and-Time attribute to ensure clock accuracy and synchronization between agent and manager. Attributes such as Power-Status would send an update on change of status and Battery-Level would send an update on crossing a prescribed level.
TABLE ॥

Typical Metric OBject ATtRIBUtes - Body WeIght

\begin{tabular}{|c|c|c|}
\hline Attribute name & Remark & Value \\
\hline Handle & $\begin{array}{l}\text { A unique reference ID } \\
\text { for this object }\end{array}$ & 1 \\
\hline Type & $\begin{array}{l}\text { The type and version } \\
\text { of the agent }\end{array}$ & $\begin{array}{l}\text { MDC_PART_SCADA MDC_ } \\
\text { MASS_BODY_ACTUAL }\end{array}$ \\
\hline Metric-Spec-Small & $\begin{array}{l}\text { Describes the } \\
\text { characteristics of the } \\
\text { observation. }\end{array}$ & Periodic, agent initiated \\
\hline Unit & Unit of the observation & $\mathrm{kg}$ \\
\hline Absolute-Time & $\begin{array}{l}\text { Timestamp of the } \\
\text { observation being } \\
\text { reported }\end{array}$ & $21 / 11 / 2015-09: 44: 15.22$ \\
\hline $\begin{array}{l}\text { Simple-Nu- } \\
\text { Observed-Value }\end{array}$ & Actual observation & 75.6 \\
\hline
\end{tabular}

Data sources are modelled as objects defined by the metric class; with sub-classes defined for sources of type; numeric, enumeration, and real-time sample arrays. Table II shows the attributes that would be used for body weight.

Every instance of an object is allocated a handle that is used as a unique reference ID for that object. This has the advantage that several objects of the same class and with otherwise identical attribute values can exist, as may be the case in a multi-channel agent such as an ECG device. The type attribute provides the context and specifies the data source. This can be further refined using other attributes, for example to specify the location of an observation or a specialized type of sensor.

\section{IEEE 11073-20601 State Model}

The IEEE 11073-20601 [9] state model (see Fig. 4) is association based, and all aspects of the exchange are negotiated between agent and manager. This includes the version of the protocol and device specialization. During association, agents offer the set of versions that they support and the manager selects a common version, thereby giving backward compatibility. After association, the agent and manager will negotiate a mutually agreed configuration of the agent that will be used by the manager for the remainder of the association. Agent and manager then enter operating state, in which observations may then be sent. Recent changes that have been made to the state model include a further state before operating to allow the status and value of the agent clock to be determined by the manager, and the agent clock set if required.

In addition to the normal state transitions shown in Fig. 4, the IEEE 11073-20601 standard includes a fully specified stateevent table to define behavior under all conditions of state and event and to ensure that agent and manager become resynchronized after all error conditions and that no data is lost.

\section{E. IEEE 11073 Nomenclature}

The nomenclature of IEEE 11073-10101 [11] is used where this exists, and new terms have been created when required for the specializations. This has largely been in the domains of strength and fitness and independent living. New terms follow 


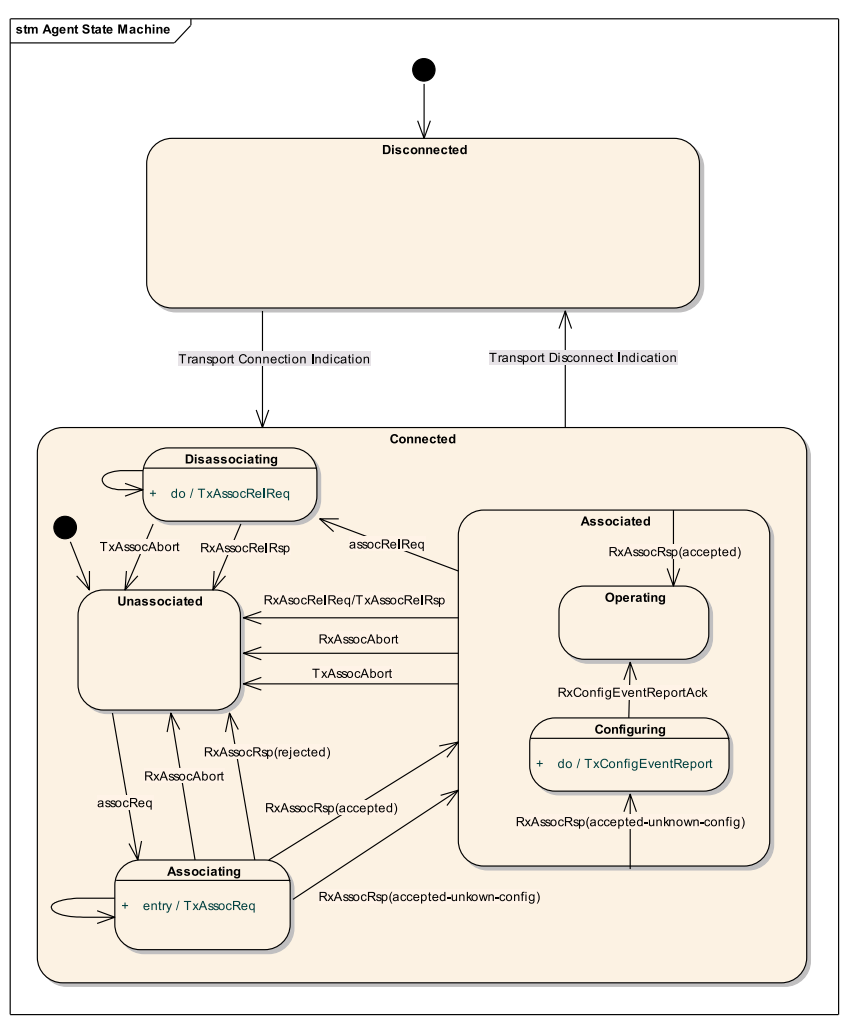

Fig. 4. IEEE 11073-20601 state model (from [9]).

the conventions of IEEE 11073-10101 and remain compatible. They will be incorporated in future revisions of IEEE 1107310101.

IEEE 11073-10101 nomenclature is defined as both a binary and a textual code (e.g., 2::57668, MDC_PART_SCADA:: MDC_MASS_BODY_ACTUAL), the binary code being used in IEEE 11073 devices for efficiency, with the corresponding textual code being used in ASCII based protocols such as HL7. Like terms are grouped into partitions, with 16 bits allocated for the term and 16 bits for the partition from which the term is taken. This results in a unique 32 bit code for each term.

\section{F. Optimization of the IEEE 11073 Protocol}

The IEEE 11073-20601 protocol contains a number of optimizations to increase efficiency and support for low resource devices.

1) Association Based Protocol: IEEE 11073 is an association based protocol. It is designed to send the configuration and initial values of attributes once on association, and thereafter will only send updates for those attributes where a value has changed. Typically this will occur when an observation is initiated in the agent and the observed value and its timestamp alone are sent. This minimizes the total information that has to be sent.

2) Nomenclature: Nomenclature is coded as numeric (binary) codes for efficiency. In addition, nomenclature is segmented within partitions, each of which will contain similar types of code (e.g., units). In this way assumptions of the partition may be made for specific attributes (e.g., type, unit) requiring only the 16 bits of the term to be sent.

3) Binary Messages: IEEE 11073 defines the structures of attributes and messages using ASN.1 and an encoding rule set is selected at association. Normally a binary encoding rule set is used as this gives the most compact form of message format. The Medical Device Encoding Rules (MDER) has been developed especially for the purposes of IEEE 11073, and is fully described in IEEE 11073-20601.

4) Configuration: IEEE 11073-20601 includes the concept of the standard configuration. If an agent is configured as a "standard device" (all attributes and their values defined in advance) then the "standard configuration" is presented as the DevConfiguration-Id in the initial association message and MDS attribute.

The standard configuration allows the manager to create the objects and attributes with initial values, without requiring the agent to send its configuration. This expedites reaching the operating state and reduces the number of messages that are sent.

Agents that do not conform to a standard configuration are referred as extended devices, and will send their configuration. On subsequent association, if the manager recalls the configuration, it can indicate to the agent that the configuration does not need to be resent, and there is a consequent saving in the number of messages.

5) Attribute-Value-Map: Typically objects will send repeated observations having the same format of content each time; usually this will be the observed value and the timestamp. Medical Device Encoding Rules (MDER) will encode this as a series of tuples that will include attribute ID, attribute value and length of the attribute value; one tuple for each attribute being reported. As the same format of message will be sent frequently and its format is known in advance, then this format can be conveyed to the manager in an attribute known as the Attribute-Value-Map. When the fixed format form of update is used, the format information (attribute ID and length for each attribute) can be omitted from the message and substituted from the Attribute-Value-Map attribute in the manager to decode the message, thereby saving this common overhead in each such message.

Table III shows a typical IEEE 11073 message in fixed format form reporting a blood pressure. The message has 60 bytes, and fits within a single ZigBee frame.

In fixed format, attributes values are reported by an object in the message using the format specified by the attribute value map for that object. This removes the need to include information in the message to identify the attribute being reported and its length. In the example of Table III, 16 bytes are saved per message, and this prevents the need to send 2 ZigBee frames, saving further ZigBee protocol overhead.

6) Multi-Component Messages: The IEEE 11073 message format is hierarchical and highly compartmentalized. This allows a message to contain many separate parts, such as multiple observations, from multiple attributes and from multiple objects that are clearly delineated.

This flexibility allows many elements to be carried in the same message, thereby removing overhead and making transmission 
TABLE III

IEEE 11073 Message RePorting BloOd Pressure

\begin{tabular}{ll}
\hline \hline Message & \multicolumn{1}{c}{ Comment } \\
\hline E700 & Type of APDU (data) \\
$003 \mathrm{E}$ & Length (62) \\
$003 \mathrm{C}$ & Length (60) \\
0002 & Invoke ID - used for confirmation \\
0101 & Confirmed event report - fixed format \\
0036 & Length (45) \\
0000 & Object handle (0) - object sending the report \\
FFFF FFFF & Event time (not used) \\
0 D1D & Scan report - fixed format \\
$002 C$ & Length (44) \\
F000 & Scan request ID (F000) \\
0000 & Scan report number (0) \\
0002 & Number of observations \\
0024 & Length (36) \\
0001 & Object handle (1) - object sending the observation \\
0012 & Length (18) \\
00030006 & Number components/Length of compound numeric \\
0080 & Systolic (128) \\
0053 & Diastolic (83) \\
0069 & Mean (105) \\
20161129 & Date \\
12512100 & Time \\
0002 & Object handle (2) - object sending the observation \\
$000 \mathrm{~A}$ & Length (10) \\
0043 & Pulse (67) \\
20161129 & Date \\
12512100 & Time \\
\hline \hline
\end{tabular}

more efficient. However it also results in a large number of length fields for a simple message. However we took advantage of these length fields and implemented strict parsing, checking for consistency that the component was exactly the specified length, thereby providing a high degree of error checking for transmission and implementation errors and this resulted in a robust implementation.

Several fields provide further checks for reliability. This includes:

1) InvokeID - used to check for arrival of the response to a request. Failure to receive a response will result in timeout.

2) Scan report number - used to check for receipt of duplicate messages.

7) The Scanner: IEEE 11073 includes the scanner as a further level of optimization when repeatedly sending observations from multiple objects in the same message. Like the attributevalue-map, the scanner specifies the format of the message to allow observations from multiple attributes from several separate objects to be multiplexed. This removes the need to include information in the message to identify the object, the attributes being reported and their lengths.

8) Real-Time Sample Array: The real-time sample array provides an optimized message structure when sending many observations from an object having a periodic sample rate, such as ECG. The observed values are sent as integer values without timestamps that are scaled and level shifted by coefficients. Time instants are given by the period of the samples.

9) Real Type: MDER defines a format for the real type that conveys both its value and precision. In place of the IEEE real type which normalizes the mantissa to the range $0.99 \ldots$ to 0.1

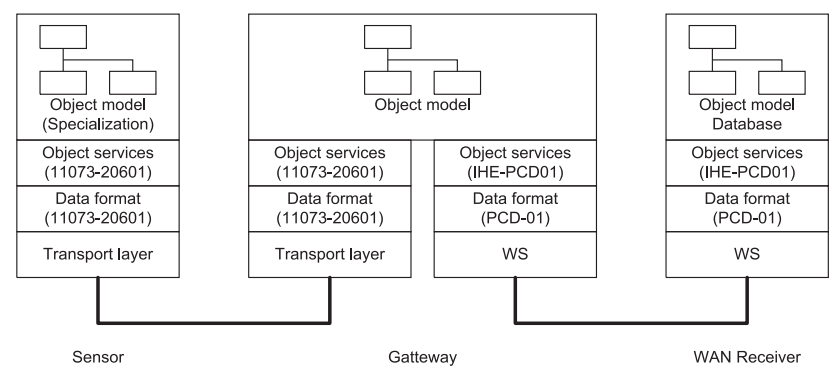

Fig. 5. Object model of IEEE 11073 mapped to IHE PCD-01.

and uses the exponent to scale to the value, MDER specifies the mantissa as the value in integer form (base 10) and uses the exponent to scale to the precision of the observation. For example, given an integer mantissa of 10 and then the precision can be differentiated by scaling as $10,10.0$, or 10.00 .

\section{G. End-to-End Integration}

As part of the development of IEEE 11073, the devices group of Integrating the Healthcare Enterprise (IHE) developed a protocol for Point of Care Devices (PCD) that would take data from a medical device following IEEE 11073 standards and place it in an HL7 message. A profile of IHE PCD-01 was defined to allow data from IEEE 11073 devices and IEEE 11073 nomenclature to be used directly through a transparent mapping of attributes and objects, and so support direct end-to-end transmission (with only a change of format in the packet in the gateway). This was accomplished by defining how values from specific attributes of the IEEE 11073 device objects are placed in specified fields of the IHE PCD-01 message and thereby support the generic gateway (see Fig. 5).

In the same way, a similar object/attribute approach may be used to define the structure of a database to accept the data from an IEEE 11073 device transparently. Thus a new type of device may be added to the platform without need to change the firmware of the gateway or the structure of the database.

\section{H. Relationship to Other Standards}

1) IHE PCD-01 and HL7 V2: The object models of IEEE 11073 allow device data to be mapped directly into messages of other standards. Standards such as IHE PCD-01 (a profile of HL7 V2) have a well-defined hierarchical format with fixed fields for specific elements of data. These elements can be filled automatically with values taken from the respective attributes of objects following a generic set of rules. Fig. 6 shows how a transaction message containing a blood pressure observation can be constructed: device information such as unique id is taken from the system object; blood pressure observation and attribute values such as unit and timestamp from the BP object; and pulse rate observation and attribute values from the pulse object. This approach is fully profiled in the Continua Design Guidelines [12].

An example of the resulting IHE PCD-01 message for the blood pressure observation of Table III is shown in Table IV, both being captured from our gateway. The example shows how each piece of information for the IHE PCD-01 message will exist as an attribute provided by the IEEE 11073 protocol. 


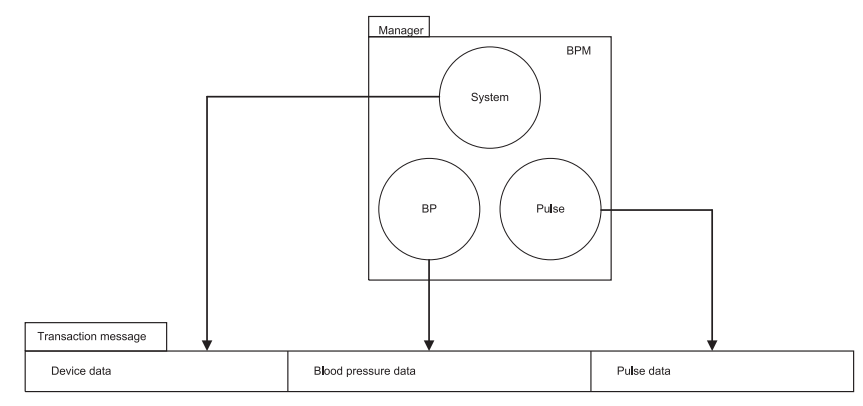

Fig. 6. Relationship to other standards.

TABLE IV

IHE PCD-01 Message Reporting Blood Pressure

\begin{tabular}{|c|c|}
\hline & Message \\
\hline 1 & 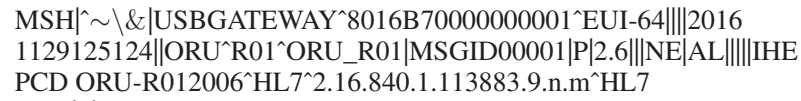 \\
\hline 2 & $\begin{array}{l}\text { OBR|1|AB12345^USBGATEWAY^8016B70000000001^EUI- } \\
\text { 64|AB12345^USBGATEWAY^8016B70000000001^EUI- } \\
64 \mid 182777000^{\wedge} \text { SNOMED-CT||20161129125121|20161129125124 }\end{array}$ \\
\hline 3 & OBX|1||528391^^MDC|1|||||||X|||||||8016B70000000003^EUI-64 \\
\hline 4 & $\begin{array}{l}\text { OBX|2|DTM|67975^MDC|1.0.0.0|20161129124952||||||R||| } \\
20161129124952\end{array}$ \\
\hline 5 & $\mathrm{OBX}|3|\left|150020^{\wedge} \mathrm{MDC}\right| 1.0 .1|||||||\mathrm{R}||| 20161129125121$ \\
\hline 6 & $\mathrm{OBX}|4| \mathrm{NM}\left|150021^{\wedge} \mathrm{MDC}\right| 1.0 .1 .1|128| 266016^{\wedge} \mathrm{MDC}|||| \mathrm{R}$ \\
\hline 7 & $\mathrm{OBX}|5| \mathrm{NM}\left|150022^{\wedge} \mathrm{MDC}\right| 1.0 .1 .2|83| 266016^{\wedge} \mathrm{MDC}|||| \mid \mathrm{R}$ \\
\hline 8 & $\mathrm{OBX}|6| \mathrm{NM}\left|150023^{\wedge} \mathrm{MDC}\right| 1.0 .1 .3|105| 266016^{\wedge} \mathrm{MDC}|||| \mid \mathrm{R}$ \\
\hline 9 & $\begin{array}{l}\mathrm{OBX}|7| \mathrm{NM}\left|149546^{\wedge} \mathrm{MDC}\right| 1.0 .0 .2|67| 264864^{\wedge} \mathrm{MDC}|||||\mathrm{R}||| \\
20161129125121\end{array}$ \\
\hline 1 & Message header giving information on the gateway \\
\hline 2 & $\begin{array}{l}\text { Header to indicate the message is an observation report and includes } \\
\text { information on the gateway and time of the message }\end{array}$ \\
\hline 3 & $\begin{array}{l}\text { Header to indicate that this is an observation report from a BP meter } \\
\text { with information of the device including its EUI- } 64\end{array}$ \\
\hline 4 & $\begin{array}{l}\text { The gateway reports the coincident timestamp as an audit of the } \\
\text { translation of timestamps by the gateway }\end{array}$ \\
\hline 5 & $\begin{array}{l}\text { The blood pressure observation. A common timestamp is given for the } \\
\text { three components of the blood pressure }\end{array}$ \\
\hline 6 & Systolic blood pressure observation \\
\hline 7 & Diastolic blood pressure observation \\
\hline 8 & Mean blood pressure observation \\
\hline 9 & Pulse rate observation with timestamp \\
\hline
\end{tabular}

Note that for security reasons we do not include patient identification in the IHE PCD-01 message, rather we report the EUI-64 (unique identifier) of the device, which is mapped to the patient in the database.

2) FHIR: Current efforts are directed towards specifying the resources that are required to support transmission of device observations within messages of the FHIR protocol. The FHIR protocol differs from IHE PCD-01 by defining hierarchical object models based on pre-defined components or resources. FHIR (as HL7) mainly addresses the transmission of clinical records; however the specific resources to support medical devices have now largely been defined [12], although efforts to capture all aspects of a device remain.

3) Generic Gateways: This highly constrained object approach, together with the plug-and-play interoperability of IEEE 11073 devices, provides a powerful technique for developing generic gateways; able to handle devices from all domains equally and without need for firmware update when new types of device are introduced.

Moreover, as the device object model contains detailed information on the device, such as its current time, operations such as correction of timestamps can be made following exact procedures [13].

HL7 and IHE have produced guidelines for management of timestamps. However these were developed with the expectation that all devices would be within the health care enterprise, where there are good and reliable network connections and devices are well managed. Moreover most devices are likely to be in close proximity and will send data as soon as it is created. It is assumed that devices will have accurate time, and issues of time zone and daylight savings are minimized.

In a practical remote patient monitoring platform, the ideal conditions of the health care enterprise do not apply. The limitations must be identified and a protocol developed to support working in such environments and this must indicate the conditions under which the platform is operating. Furthermore the protocol must allow devices to continue to operate in any adverse conditions that may prevail. This will include the gateway being unable to acquire accurate time as not all mobile (cell) phone networks provide network time on registration to the network, and the NTP server may not be accessible. In some circumstances, it may be more important to send the data than have the correct timestamp. In such cases the data will be sent, but we must ensure that the reliability of the timestamp is indicated in the message.

This was accomplished by including a coincident timestamp to provide the correlation between the respective clocks of the device and gateway, a full analysis of timestamp requirements is given in [13] and the approach has been validated in our implementation. Further lessons remain to be learnt to fully define all operating conditions of agent and manager.

4) Integration With and Adoption by Other Protocols: The IEEE 11073 standards provide a high level and wellstructured object description of a device. Moreover, the open consensus approach of the IEEE for standards development results in wide agreement of the standard for a device. There is therefore merit in other protocols following the approach of IEEE 11073, including the device models and its nomenclature. Not only does this result in better device implementation, but use of closer semantics can simplify later integration of data in a mixed technology environment.

This approach has been largely adopted by the BT Medical Device Special Interest Group (BT MED SIG) in developing the profiles for medical devices that follow BT LE, with the intention that IEEE 11073 and BT LE devices can be integrated on the gateway at the object level. (Note that, in contrast, BT LE defines differently the format of the notification (observation) in each device profile, necessitating update of the gateway firmware with each new device).

\section{Changes to the Baseline Standard}

ZigBee Pro [14], upon which the ZigBee Health Care Profile is based, has a fully acknowledged application layer protocol 


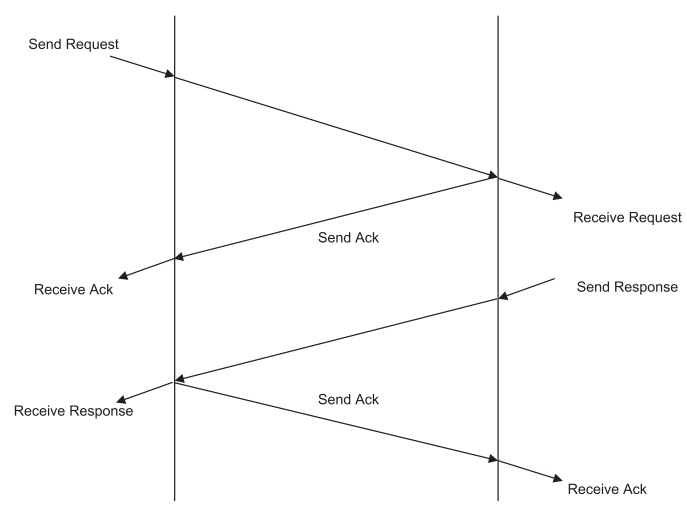

Fig. 7. Normal ZigBee application layer protocol.

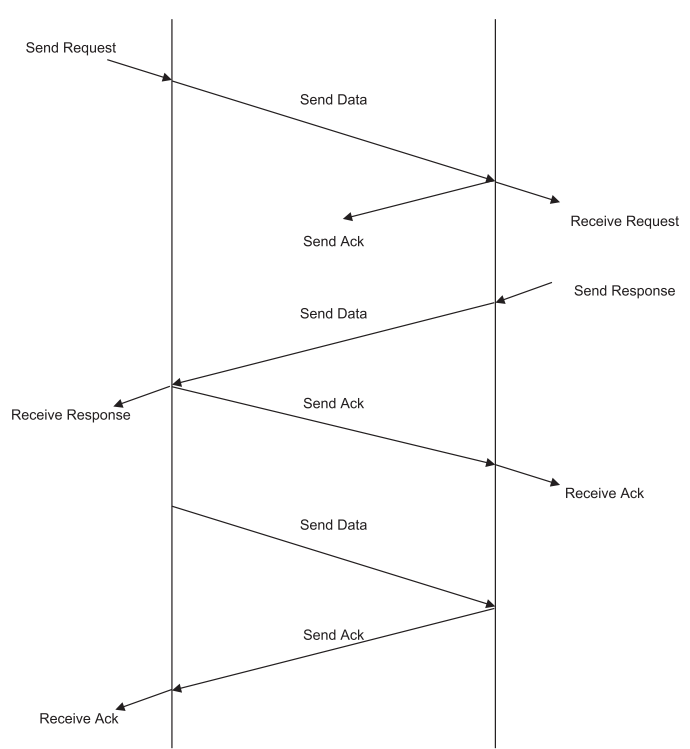

Fig. 8. ZigBee transport layer protocol with delayed ACK.

that provides a separate acknowledgement of correct transmission of a frame and that is passed as an indication to the application; the so called Application support sub-layer (APS) acknowledgement. This acts as both a mechanism to ensure reliable transmission (the ACK is only received after correct transmission and any retransmission) and provides application layer flow control.

The application layer of the IEEE 11073 protocol is largely based on request - response to confirm actions. This results in a modified exchange of packets for an application layer protocol that has an explicit application layer ACK. As seen in Fig. 7, the normal behavior is to receive the application layer ACK, followed by the application layer response.

However a problem arises if the transport layer acknowledge for the request is lost in transit - the request arrives and the response is sent and received, but the response is received in advance of the application layer ACK for the request, which is resent on receipt of a retransmission of the request. The situation is shown in Fig. 8.

The explicit ACK of the ZigBee application layer gives rise to several variations from the standard state model of IEEE

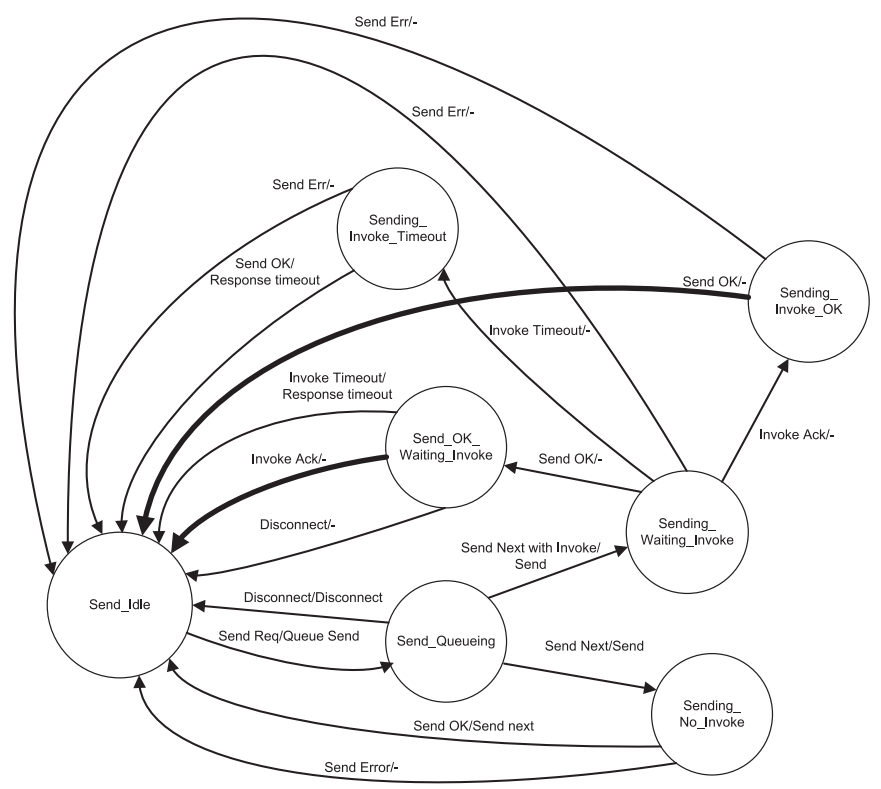

Fig. 9. Amended state model of IEEE 11073 for use with ZigBee transport layer protocol.

11073 that must be handled by a ZigBee implementation (and any other with explicit transport layer ACK). The full set of variations includes:

1) Request acknowledge arrives in advance of data response (normal)

2) Request acknowledge arrives but no data response sent (normal timeout)

3) Data response arrives in advance of request ac knowledge (retransmit of data request - otherwise normal)

4) Request acknowledge arrives but data response lost (response send error- will result in network disconnect)

5) Data response arrives but no request acknowledge (request send error - will result in network disconnect)

6) Data response timeout and no request acknowledgement (request send error - will result in network disconnect)

7) Data response arrives but response acknowledgement lost (response send error - will result in network disconnect)

Each of these extra conditions must be added to the normal state model of IEEE 11073 in order that a device will handle all variants and error conditions correctly. The modified state diagram is shown in Fig. 9 (the explicit disconnect event is omitted in most cases in the diagram for clarity).

There will be further complications when the acknowledgement to a response is lost, as a device may receive the next request before the acknowledgement to the previous response is received. Not only must the response to the new request not be sent until the acknowledgement to the previous response is received, but there are implications for the need for buffers, as both responses will be created in memory.

Note that it is necessary to wait for the duration of the timeout for the response before moving to the unassociated state when a send error is indicated for the request, as it may be the case that the request has been received (the ACK is being lost) and a response is being sent and may be received with a delay. This 


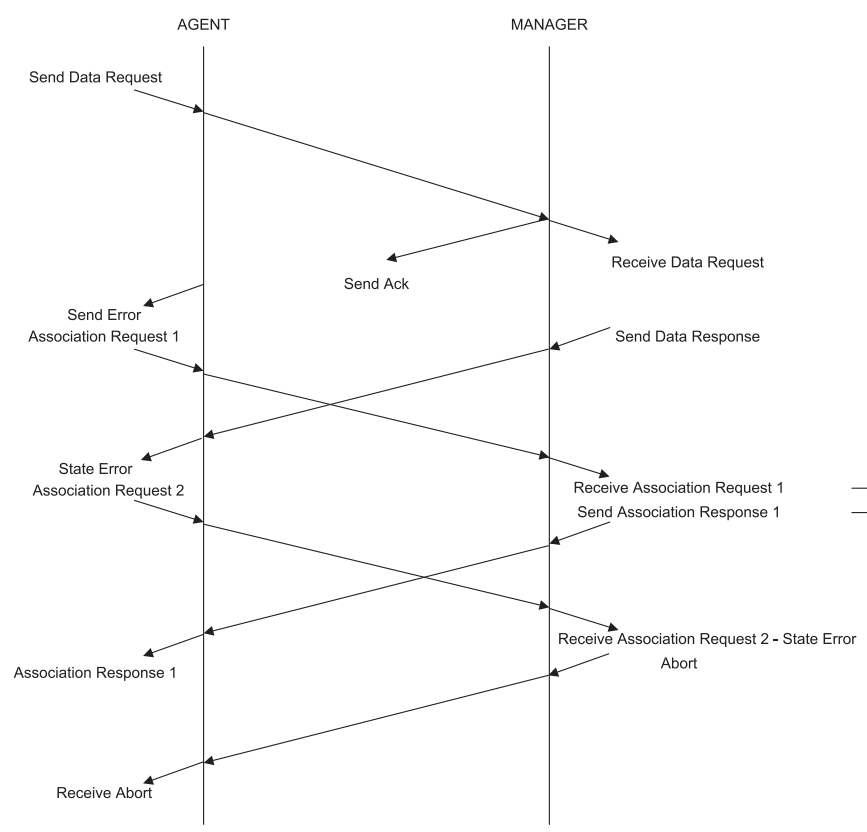

Fig. 10. Protocol issues following send error and the need for a timeout wait state.

response must be ignored and only the send error indicated to the higher application.

Failing to wait for this timeout and reporting the send error immediately can result in a serious problem; if a new association request is sent immediately as a result of the send error (effectively a disconnect-connect), then the incoming delayed response will be interpreted incorrectly as the response to the association request and a state error will be indicated, Fig. 10.

The problem perpetuates if a second association request is sent immediately, as the incoming association response to association request 1 will be interpreted as the response the association request 2. Moreover, association request 2 will be delivered to the manager when it is in the associated state, and so a state error is indicated and the manager will send an abort. If not handled correctly, agent and manager can enter a perpetual cycle.

This timing issue was observed in our implementation and has since been addressed in later versions of the IEEE 11073-20601 standard, with changes to the state model to include the timeout wait state following an error condition and after an abort is sent.

\section{The ZigBee Health Care Profile}

\section{A. The ZigBee Protocol}

ZigBee has been developed as a low power, highly functional wireless technology that is capable of forming a mesh network. It is based on IEEE 802.15.4 as link layer (MAC) and adds: network layer for routing; application support sub-layer (APS) for reliability and support of higher functionality; management and security; ZigBee device objects (ZDO); and ZigBee clusters (see Fig. 11).

The functionality of the ZigBee clusters may be used to complement IEEE 11073 to provide management of devices, including provisioning, Over the Air Update (OTAU), security

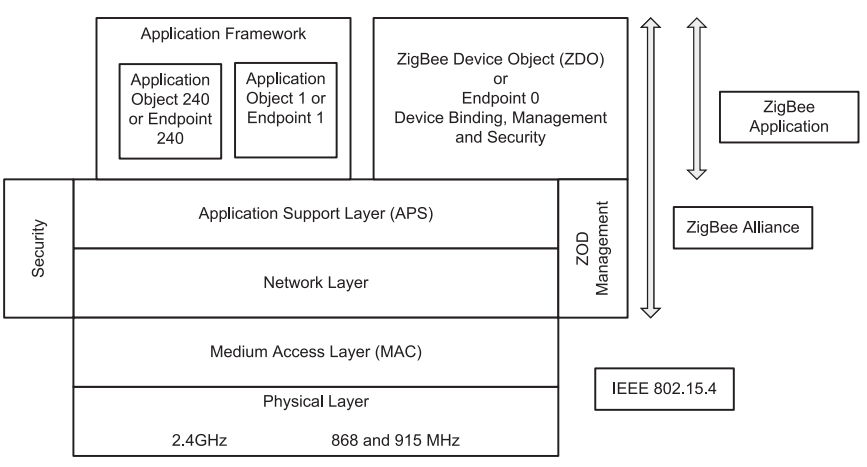

Fig. 11. ZigBee layers.

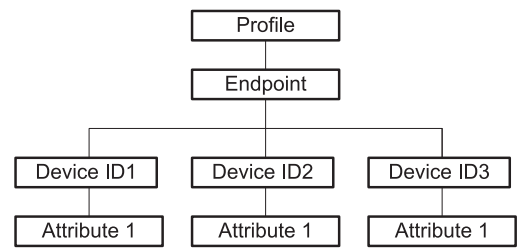

Fig. 12. ZigBee profile and its components.

and debugging. ZigBee also supports device discovery and announcements when a device joins a network.

ZigBee also includes robust security, using AES for network layer encryption, and application layer security to support exchange of network key (randomly generated) between the security center of the network and an authenticated device joining the network.

The IEEE 11073 protocol is supported transparently through use of a tunnel. The tunnel is configured as a ZigBee cluster with attributes to designate state, and with cluster commands to perform connect, disconnect and transfer Application Protocol Data Units (APDU) transparently. Each tunnel is advertised through provision of the tunnel cluster on a configured endpoint (see Fig. 12).

APDU transfers of less than 240 bytes are handled by the normal ZigBee transport layer mechanisms, using the reliable transfer method with acknowledgement of each 80 byte fragment, and with the APDU being passed from the transport layer to the application when complete. This is sufficient to support most simple agents that have a small number of objects in the configuration; the configuration report being the largest single APDU.

For larger APDUs, the partition cluster is used. This has the advantage of transferring the APDU as a series of smaller frames using the normal ZigBee transport layer mechanism with its limited buffer space, and allows the larger APDU to be reconstructed in an appropriate sized buffer allocated from application memory space on notification of the incoming APDU.

The specification of the clusters, attributes, configuration and security model are given in the ZigBee Health Care Profile [14].

\section{B. Low Power Operation}

The ZigBee protocol exploits the concept of the "sleepy" end-device to achieve ultra-low power operation. In this mode, 


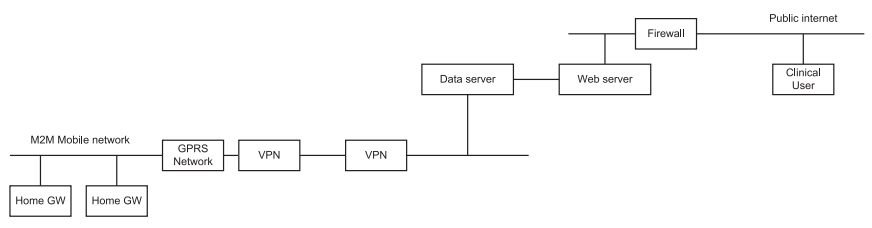

Fig. 13. Secure end-to-end M2M solution.

the "sleepy" end-device switches off its receiver and may also switch off its processor for significant periods of time (in the order of seconds) to consume microwatts of power in place of tens of milliwatts. The "sleepy" end-device wakes momentarily to make an enquiry to its local router if any frames are awaiting delivery. With a negative answer, the "sleepy" end-device immediately resumes sleep, otherwise the frame is delivered and processed; the process of enquiry lasting tens of microseconds. Such a cycle leads to extremely low power, but has the advantage that the "sleepy" end-device appears to be constantly connected to the network, and the IEEE 11073 protocol remains associated. In our platform, using a sleep cycle of 7.5 seconds; sensors remained associated for months using AA size batteries. This approach has the further benefit that loss of connection of a sensor from the network can be detected and the application layer will generate an explicit message to the application, which can maintain complete status of each device and take appropriate action (e.g., attempt to reconnect, report the error).

\section{A Secure End-TO-End PlatForm}

The end-to-end platform that we have implemented uses a Machine to Machine (M2M) solution to provide wide area network connectivity and deliver a secure system. In the M2M solution as shown Fig. 13, the M2M provider establishes a secure private network within the mobile infrastructure of a mobile (cell) operator through provision of its own APN. A Virtual Private Network (VPN) is then established between the M2M provider and the data server to create a single secure private network between each home gateway and data server.

Within the M2M mobile network, the home gateways are authenticated on the network through the SIM card (which may be soldered to the board to give physical security). Moreover, the home gateways (as a private network) may each be given a static IP address, thereby supporting direct access compared to a dynamically allocated address and having need of a separate registration server to map a static identification to current IP address.

A clinician portal runs as an application on the web server and has direct access to the data server. The firewall provides secure access to the web server from the public internet.

\section{Clinical Trials With the Platform}

Our platform was first implemented in the Hydra project, which had as goal to demonstrate that health data could be transported as part of the communication within a smart meter infrastructure. The communication standard for smart meters was to include ZigBee, and the Hydra project demonstrated

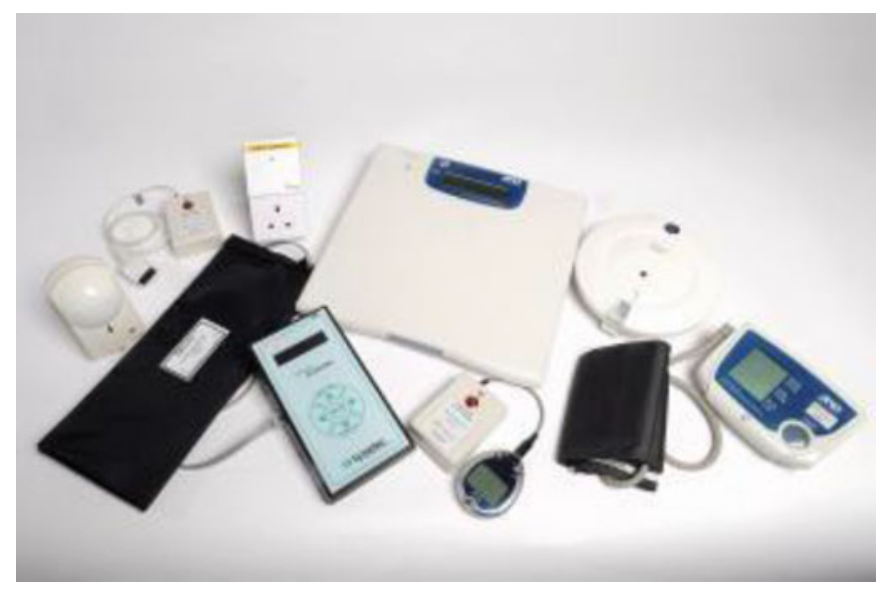

Fig. 14. Home gateway and seven devices of the Hydra, inCasa and reaction projects.

data from patient homes being transferred through the communication infrastructure of the Echelon smart meter [15].

The Echelon smart meter included an uncommitted slot that could be used to transfer small packets of data transparently using powerline communication to a data concentrator in the local vicinity (power sub-station) and then through any wide area network, such as GPRS. In the Hydra project, a ZigBee module was placed in the uncommitted slot and communicated using ZigBee with sensors placed in the home.

The goal of the Hydra project was extended, and a purpose home gateway was designed and developed that sent data using its own GPRS modem directly to the data server. In addition, further sensors were developed to demonstrate the interoperability between sensors for health (e.g., blood pressure, weight) and ambient assisted living (e.g., PIR, bed/chair sensor).

Our approach for integration followed that of others [16], [17], and interfaced to the internal signals of commercial off-the-shelf devices where these were available. A wireless module was purpose designed to be compatible with the signals and footprint of the wireless module of the A\&D blood pressure meter and A\&D weighing scale. Uncommitted pins of the internal connector were used to extend the functionality of the module to support use in other devices. We further modified the module to include a bi-colored LED (red/green) that was used to provide indication to the use of connect/disconnect with the network, when a measurement was being taken, and when sent correctly. An LED was retro-fitted to the A\&D devices as these did not provide status indication to the user.

Seven separate wireless sensors were developed for various devices (blood pressure, weight, pulse oximeter, blood glucose meter, PIR, bed/chair sensor, medication dispenser) to demonstrate interoperability and to investigate the advantage of such a platform (see Fig. 14).

We developed our own pulse oximeter as we considered current commercial devices too small and difficult to operate by frail elderly. Operation was simple; place the finger probe, press the large button, wait for the green light, remove the probe.

We developed a base station for the blood glucose meter, which was connected through a serial cable to read out the data. 


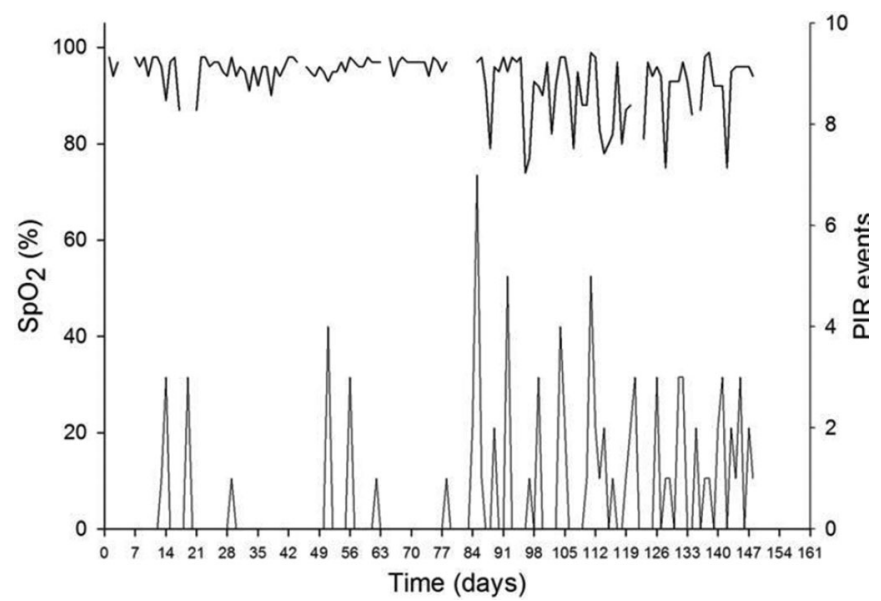

Fig. 15. Night-time PIR motion data (lower curve) and $\mathrm{SpO}_{2}$ data (upper curve) recorded over a 21-week period in a patient with COPD. An exacerbation occurring about midway through the recording period was associated with increased night-time activity and a reduced $\mathrm{SpO}_{2}$ level (from [20]).

We have since integrated a smoke detector, and similar devices from other manufactures, such as pediatric weigh scales, all without change to the firmware of the home gateway, to demonstrate the flexibility and expandability of the system.

In the inCasa project, frail elderly patients with chronic disease, including congestive heart failure (CHF) and chronic obstructive pulmonary disease (COPD), were given health sensors to monitor their disease and ambient assisted living sensors to monitor their frailty. Data was monitored daily to determine any changes significant of exacerbation of the disease or unusual changes of activities of daily living (ADL) [18], [19].

In the Reaction project, all patients registered with a general practice in Chorleywood, UK, were provided with a monitoring kit comprising the gateway, blood pressure meter and blood glucose meter. All patients were taught how to use the devices in the clinic and instructed to take the kit home with them and take daily measurements for a period of two weeks. Data was reviewed at the end of the two week period to determine if the condition was managed appropriately or not. Those patients deemed well managed were asked to return the kit to the clinic, after which it was passed to the next patient. Otherwise the patient was instructed to make an appointment to see the clinician to review the data and asked to continue taking daily measurements.

\section{REsults}

\section{A. InCasa}

Thirty patients were monitored and several exacerbations were noted. We examined both sets of data, health and activities of daily living, to determine in which data set(s) there were changes and if changes in the two data sets were correlated, and were correlated with clinical events. Two examples are presented.

Fig. 15 shows the night time PIR motion data (lower curve) recorded over a 21 week period, together with the $\mathrm{SpO}_{2}$ data

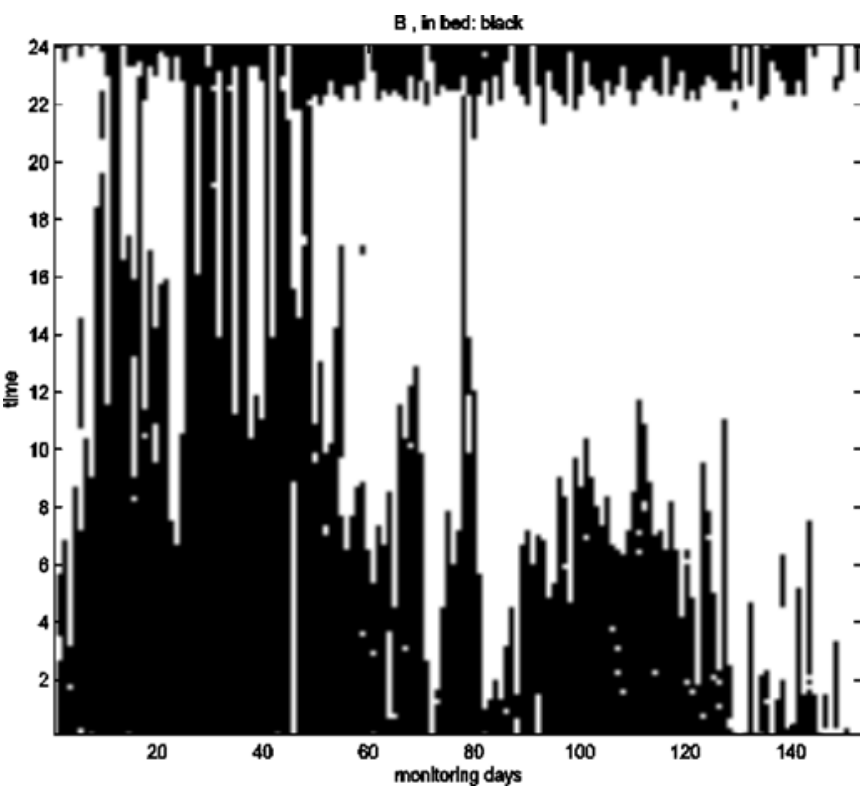

Fig. 16. Bed occupancy (from [14]).

(upper curve) for the same period for a patient with COPD. An exacerbation occurring about midway through the recording period was associated with increased night-time activity and a reduced $\mathrm{SpO}_{2}$ level [20].

Fig. 16 shows the time in bed for a patient with COPD over a 21 week period. Each vertical column shows bed occupancy for a 24 hour period, with midnight at the bottom. Black shows a period in bed, and white when not in bed [14].

The data shows that the patient goes to bed at around 23.30 each day, but there are several trends where the time in bed reduces. Examples include day 10 to 20,30 to 70 , and 100 to 130. The first two episodes correspond to exacerbation and with intervention the patient recovers and returns to a normal sleep pattern. The final episode corresponds to death.

\section{B. Reaction}

One hundred and sixty patients were asked to undertake a period monitoring using the kit on at least two separate occasions that corresponded with their routine 6 monthly diabetes review. As part of the project, we purposely designed the gateway to be unobtrusive (no user interface) and the devices to be simple in operation. This allowed the patients to be instructed how to use the sensors in the clinic on their first visit, with a refresh on subsequent visits if requested. Most patients did not ask for a refresh. In $97 \%$ of cases, the patient used the kit at home without any further contact with the health center for advice, proving the concept and design.

Of those that contacted the health center, the major problems were:

1) Confusion over use of the glucose strips; typically patients would put the blood on the strip before inserting the strip in the meter.

2) Re-site the gateway in a better position within the home to receive GPRS signal.

3) Reposition the blood pressure cuff on the arm. 


\section{DISCUSSION}

\section{A. Standards}

The IEEE 11073 PHD standards are designed to be independent of the transport layer, and provide a specification of the generic characteristics that any transport layer must possess to be used with the standards.

A profile of ZigBee, ZHCP, has been specified to support IEEE 11073. However the ZigBee protocol has a number of characteristics that were not anticipated in the development of the IEEE 11073-20601 standard and these have required us to develop a number of modifications to the state model.

Specifically, the explicit ACK of the application layer (that may be used for flow control and error control) had to be incorporated into a modified state model for the request and response in order to ensure correct handling of the case of a lost ACK when the associated response could be received before the ACK, or of a lost ACK and a send error had to be notified.

Although technically challenging, the explicit ACK of ZigBee results in a more reliable protocol.

Using ZigBee confers several technological advantages over other radio technologies:

1) Use of IEEE 802.15.4 as link layer in ZigBee and the sleepy end device protocol leads to ultra-low power, whilst devices remain connected to the network. In our devices we used a sleep period of 7.5 seconds. This gave a reasonable response time, allowed use of the watchdog timer (typically maximum 8 seconds) and gave very low power.

2) The number of devices on a network is practically unlimited; IEEE 802.15.4 uses a short address format of 16 bits giving 65535 devices. In the inCasa project, up to 4 sensors were deployed to a patient to prove concept. Typically the number is constrained by memory requirements.

3) ZigBee automatically forms a mesh network allowing simple extension within the home.

4) IEEE 802.15.4 uses a robust modulation and low data rate, thereby giving excellent range and reliable performance within a domestic environment.

Our implementation has identified several issues with the IEEE 11073 protocol and state model, and this has resulted in modifications that have been incorporated in amendments and revisions of the IEEE 11073-20601 standard. These include:

1) Extra states to allow for lost ACK.

2) Explicit timeout attribute to support sleepy end device.

3) Serialization of start-up procedure to operating state.

4) New time format and improved management of timestamps.

\section{B. Application to an Integrated Care Platform}

In addition, we have validated and demonstrated the advantages of the IEEE 11073 standards in both domains of health and telecare:

1) Physiological and assisted living sensors were deployed using a single gateway and demonstrated semantic interoperability - there was no distinction between them at the gateway. This addresses a current lack of research in this area [20], [21].

2) New types of sensors were added to the platform without change of software on the gateway, validating the plugand-play protocol of the standards.

3) Lessons were learnt regarding the requirements for timestamp management [13].

Reports to date on the implementation of IEEE 11073 on ZHCP are few, and remain restricted to concept design [15] and prototype implementation [17], and do not describe or report the changes that must be made to the generic IEEE 11073 state models.

Outcome from the inCasa and Reaction projects demonstrated the advantages of the IEEE 11073 standards. Specifically:

1) Data from physiological and ambient assisted living sensors could be collected together using a single platform with data having semantic interoperability.

2) A simple to use platform could be developed and used by patients.

3) The platform was flexible and extensible, allowing sensors to be added as needed to monitor even the most complex patient.

4) The data collected enables us to investigate changes in physiological and habits data, and whether these are associated with clinical events.

5) The kit could be deployed by the patient without the need to visit the home to install the gateway and educate the patient on the use of the sensors; visits to the home to install and remove equipment are one of the major costs of home remote monitoring.

6) Simple sensors and a gateway with no user interface reduced education time for patients, further reducing cost to deploy the system.

7) Moreover, by frequent re-use of the kit, the cost is much reduced, as fewer kits are required. In this case, 20 kits were sufficient for the 160 patients in the Reaction project, even allowing for extended periods of monitoring beyond the initial 2 week period in some cases.

\section{Future Developments}

This paper confirms that ultra-low power wireless protocols, such as ZigBee, can support IEEE 11073 with significant advantage. However technology continues to develop and will have influence on the standards. BlueTooth have recently released BT 5.0 [22], which is to include routing, greater range, larger baseband frames, and the delivery of frames to a sleepy end device.

One further development is Thread [23], also based on 802.15.4, and is a profile of 6LoPan (low power IPv6 in resource constrained networks). Adoption of IP based transport layer technology could lead to unification of technologies, including Wi-Fi (802.11); interoperability would be based on bridging and routing IP networks; managers could be in the cloud. IEEE 11073 could be implemented directly on this transport layer. 
Internet of Things (IoT) is one of the most recent developments; and seeks to include health and ambient assisted living in its remit (Healthy IoT). Although there is convergence of lower layers, the upper layers, data models and nomenclature remain to be defined. IEEE 11073 offers much to this initiative.

\section{CONCLUSION}

The IEEE 11073 PHD standards are designed to provide plugand-play semantic interoperability across the domains of health and ambient assisted living. Furthermore, the IEEE 1107320601 standard specifies a protocol that is designed to be reliable and is independent of transport layer. Other standards such as IHE PCD-01 and the guidelines from Continua profile the use of IEEE 11073 to provide plug-and-play end-to-end interoperability. This paper describes an implementation of these standards using the ZigBee Health Care Profile as the transport layer that validates the concepts.

ZigBee was found to have certain technical differences from other transport technologies. Changes to the state model of IEEE 11073 were required to accommodate but, given these modifications, $\mathrm{ZigBee}$ was found to be reliable in all respects, and gave extremely good performance as a wireless technology in terms of range, power, number of sensors connected concurrently, types of sensors, and that sensors were always connected.

The plug-and-play properties of IEEE 11073 were validated through the addition of further sensors during several projects and deployed to patients with complex needs without need for change of software in the gateway or design of the database. The inCasa project demonstrated semantic interoperability across the health and ambient assisted living domains, and the value of having both types of sensor to monitor frail elderly patients.

\section{ACKNOWLEDGMENTS}

This family of standards is the result of the work of the IEEE Personal Health Devices (PHD) working group and the author acknowledges the contribution of each of the members of the group towards the standards.

The Hydra project was funded by the Technology Strategy Board (TSB) and EPSRC, UK.

The inCasa project was funded by the European Commission.

The Reaction project was funded by the European Commission.

\section{REFERENCES}

[1] A. Darkins et al., "Care coordination/home telehealth: The systematic implementation of health informatics, home telehealth, and disease management to support the care of veteran patients with chronic conditions," Telemed. E-Health, vol. 14, pp. 1118-1126, Dec. 2008.
[2] J. G. Cleland et al., "Noninvasive home telemonitoring for patients with heart failure at high risk of recurrent admission and death: The transeuropean network-home-care management system (TEN-HMS) study," $J$. Amer. College Cardiol., vol. 45, pp. 1654-1664, May 2005.

[3] J. Fursse, M. Clarke, and R. Jones, "Early experiences of the use of remote patient monitoring for the long term management of chronic disease," $J$. Telemed. Telecare, vol. 14, pp. 122-124, 2008.

[4] J. Yao, R. Schmitz, and S. Warren, "A wearable point-of-care system for home use that incorporates plug-and-play and wireless standards," IEEE Trans. Inf. Technol. Biomed., vol. 9, no. 3, pp. 363-371, Sep. 2005.

[5] "Integrating the Healthcare Enterprise IHE Patient Care Device (PCD) Technical Framework Volume 2." [Online]. Available: http://www.ihe.net/ uploadedFiles/Documents/PCD/IHE_PCD_TF_Vol2.pdf (last accessed 19-02-2014).

[6] "HL7 messaging standard version 2.6." [Online]. Available: http://www. hl7.org/implement/standards/product_brief.cfm?product_id $=145$. Accessed on: Feb. 19, 2014.

[7] M. M. Hassan et al., "A multimedia healthcare data sharing approach through cloud-based body area network," Future Gener. Comput. Syst., vol. 66, pp. 48-58, Jan. 2016.

[8] A. Rahmani et al., "Exploiting smart e-health gateways at the edge of healthcare internet-of-things: A fog computing approach," Future Gener. Comput. Syst., 2017. [Online]. Available: https://doi.org/10.1016/ j.future.2017.02.014

[9] Health Informatics-Personal Health Device CommunicationApplication Profile-Optimized Exchange Protocol, ISO/IEEE 1107320601, 2014.

[10] "Continue health alliance design guidelines." [Online]. Available: http://www.continuaalliance.org/products/design-guidelines

[11] Health Informatics - Point-of-Care Medical Device Communication Part 10101: Nomenclature, ISO/IEEE 11073-10101, 2004.

[12] [Online]. Available: http://build.fhir.org/devicemetric.html

[13] M. Clarke et al., "Designing robust and reliable timestamps for remote patient monitoring," J. Biomed. Health Inf., vol. 19, no. 5, pp. 1718-1723, 2015.

[14] [Online]. Available: http://zigbee.org/Standards/ZigBeeHealthCare/ Overview.aspx

[15] M. Clarke et al., "Building point of care health technologies on the IEEE 11073 health device standards," in Proc. 2013 IEEE Point-of-Care Healthcare Technol., 2013, pp. 117-119.

[16] J. Kim and O. Song, "ISO/IEEE 11073 interoperability for personal health devices based on ZigBee healthcare service," in Proc. IEEE Int. Conf. Consumer Electron., 2015, pp. 263-264.

[17] Y.-F. Lee, "An interoperability solution for legacy healthcare devices," IT Prof., vol. 17, no. 1, pp. 51-57, 2015.

[18] M. Clarke et al., "Dynamic threshold analysis of daily oxygen saturation for improved management of COPD patients," J. Biomed. Health Inf., vol. 20, no. 5, pp. 1352-1360, 2016.

[19] J. de Folter et al., "Designing effective visualizations of habits data to aid clinical decision making," BMC Med. Inf. Decis. Making, vol. 14, 2014, Art. no. 102

[20] M. Clarke, "The need for an integrated approach to remote monitoring of physiological data and activity data," J. Telemed. Telecare, vol. 20, no. 3, pp. 159-160, Apr. 2014.

[21] H. Gokalp and M. Clarke, "Monitoring activities of daily living of the elderly and the potential for its use in telecare and telehealth: A review," Telemed. E-Health, vol. 19, no. 12, pp. 910-923, Dec. 2013.

[22] BlueTooth 5.0. [Online]. Available: https://www.bluetooth.com/specifi cations/bluetooth-core-specification/bluetooth5?_cldee $=$ bWFsY29sbS5 jbGFya2VAYnJ1bmVsLmFjLnVr\&recipientid=contact-d8f0778ed745 e2118be878e3b5102e43-1af7b2b3d7e84b93bdbcca4da8b35a96\&utm_ source $=$ ClickDimensions\&utm_medium $=$ email\&utm_campaign $=$ Tech\%20Updates\%20-\%202015\&esid=54da7693-49c1-e611-80f65065f38a5ba1

[23] [Online]. Available: http://threadgroup.org/ThreadSpec 